\title{
A Review on Level of Specific Absorption Rate Due to High Power Transmission Lines: Analysis toward Human Position Posture
}

\author{
Z. Ghazali ${ }^{1, *}, M . H . \mathrm{Mat}^{2}, H . A$. Rahim $^{3}$, and $A$. Syafiq $^{1}$ \\ ${ }^{1}$ School of Electrical System, UniMAP, Perlis, Malaysia \\ ${ }^{2}$ Center for Diploma Studies, UniMAP, Perlis, Malaysia \\ ${ }^{3}$ School of Computer and Communication, UniMAP, Perlis, Malaysia
}

\begin{abstract}
The main contribution of this project is the development of a homogeneous model of a man to presents the specific absorption rate (SAR) due to high power transmission line. As a low frequency application under high power transmission line of $50 \mathrm{~Hz}$ in electrical engineering, to studies the influence of human's posture on specific absorption rate. This project designs two types of human body which one design uses most cylinder block and another design use brick block where both blocks have a different value of mesh cells. For each design has four types of posture are standing, sitting, arms up and arms out by using Computer Simulation Technology (CST) Studio Software. This analysis does toward for four types of the human position postures because each posture has different value of specific absorption rate (SAR) based on the size of the mesh cells of the design. Based on two designs of the human body, the lowest of the mesh cells value will reduce time to simulate SAR. For each posture has different value of SAR for each part of the human body because the whole human body has different types of tissues. Therefore, the CST studio software uses extremely to simulate the SAR value toward human position posture due to high power transmission line.
\end{abstract}

\section{Introduction}

This project discusses about level of specific absorption rate (SAR) due to high power transmission line in Malaysia where uses $50 \mathrm{~Hz}$ of frequency to simulate the SAR value toward human body. The International Commission on Non-Ionizing Radiation Protection (ICNIRP) and the Council for European Union recommended permissible limit to exposure of electric shown in Table 1.

\footnotetext{
* Corresponding author: zulaikha_ghazali@yahoo.com
} 
Table 1. ICNIRP limit and the Council for European Union exposure limit [1].

\begin{tabular}{|c|c|}
\hline \multirow{2}{*}{ Power line operating frequency } & $\mathbf{5 0 ~ H z}$ \\
\cline { 2 - 2 } & Electric field (V/m) \\
\hline Public exposure limit & 5000 \\
\hline Occupational exposure limit & 10000 \\
\hline
\end{tabular}

This analysis was done toward on four types of the human position postures which is standing, sitting, arms up and arms out posture. Each posture has different SAR value for each part of the human body because the whole human body have different types of tissues [2]. The ICNIRP have defined SAR with unit watt per kilogram $(\mathrm{W} / \mathrm{Kg})$ and reference level of field strength from exposure to EMF to protect workers and public avoid health effects [3-5]. SAR is the quantity of how much power of an electromagnetic wave is absorbed per unit mass by an absorber such as a human body. In other hand, SAR also means a measure of the rate at which energy is absorbed by the human body when exposed to a radio frequency (RF) electromagnetic field. SAR is derived from the square of the (direct) electric field strength (E) in tissue [6]:

$$
S A R=\frac{P_{a b}}{\rho}=\frac{\sigma E^{2}}{2 \rho}=\frac{J^{2}}{2 \rho \sigma}
$$

where $\mathrm{P}$ is the power loss density, $\mathrm{E}$ is electric field strength, $\mathrm{J}$ is the current density, $\sigma$ is conductivity $(\mathrm{S} / \mathrm{m})$ and $\rho$ is the density $\left(\mathrm{Kg} / \mathrm{m}^{3}\right)$ Of the tissue interest [7-9]. For frequencies up to a few $\mathrm{GHz}$, as applied in wireless communications, SAR is normally averaged over either 1 or $10 \mathrm{~g}$. American National Standard Institute (ANSI) and Federal Communication Commission (FCC) has determine the SAR limit to $1.6 \mathrm{~W} / \mathrm{Kg}$ per $1 \mathrm{~g}$ of tissues. ICNIRP and IEEE has impose the maximum of $2 \mathrm{~W} / \mathrm{Kg}$ absorbed per $10 \mathrm{~g}$ of tissue $[10,11]$.

\section{Numerical study}

\subsection{Power transmission lines.}

Electromagnetic fields (EMF) are generated in the vicinity of power transmission lines [12]. Now, the places use the $50 \mathrm{~Hz}$ frequency tend to use 220-240 V and for $60 \mathrm{~Hz}$ tend to use $100-127 \mathrm{~V}[2,13,14]$. At extremely low frequencies (ELF) the radiating field of a source is negligible. In practical exposure situations, radiation is absolutely negligible in the ELF range. Radiation only becomes dominants at distances that are large compared to the wavelength. In the free space the wavelength is related to the frequency by the formula,

$$
\lambda=\frac{c}{f}
$$

where $\lambda$ is wavelength $(\mathrm{km}), \mathrm{c}$ is the speed of lights $\left(3 \times 10^{8} \mathrm{~m} / \mathrm{s}\right)$ and $\mathrm{f}$ is frequency $(50 \mathrm{~Hz})$. At $50 \mathrm{~Hz}$, the wavelength is very long, $6000 \mathrm{~km}$. At $60 \mathrm{~Hz}$, the wavelength is very long, $5000 \mathrm{~km}$. As a conclusion, the smaller value of the frequency, the harder of the simulation cause the wavelength become larger with higher mesh cell [15]. 


\subsection{Simulate specific absorption rate (SAR) using Computer Aided Design (CAD) software.}

Power absorption under the whole body resonant conditions is also strongly dependent on the posture of the human body. For this simulation has developed human body models for any posture based on a CAD software product. This software includes a statistical data set of anthropomorphic dimensions and can change the posture of various human bodies. After the posture of the human bodies is modified, a newly developed software module outputs the voxel data with any voxel resolution. The models described so far in this section are homogeneous because only the outline of the human bodies can be obtained with the CAD software. This is a significant shortcoming because an internal inhomogeneous structure can affect electromagnetic power absorption in the human body [16].

The FDTD method was used to calculate Whole Body Averaged Specific Absorption Rate (WBASAR). The change of the postures from arms down to arms up increased the WBASAR at most of frequencies of interest. SAR increase due to raising arms was more dominant at low frequencies and in the older models [17]. A change in the posture of the human body can significantly affect the way in which it absorbs radio frequency EMFs [8].

\subsection{Hugo model simulate with poser software and manipulate in MATLAB.}

In order to perform electromagnetic simulations, the dielectric properties allocated to various tissues and organs in the frequency ranges should be concerned. The properties of Hugo model's tissues and organs at $50 \mathrm{~Hz}$ frequency which will be used for skin refer to Table 2. While Figure 1 show various type of posture of human body.

Table 2. The dielectric properties of Hugo model tissue at $50 \mathrm{~Hz}[18]$.

\begin{tabular}{|c|c|}
\hline Relative permittivity & Conductivity (S/m) \\
\hline 1136 & 0.00020 \\
\hline
\end{tabular}

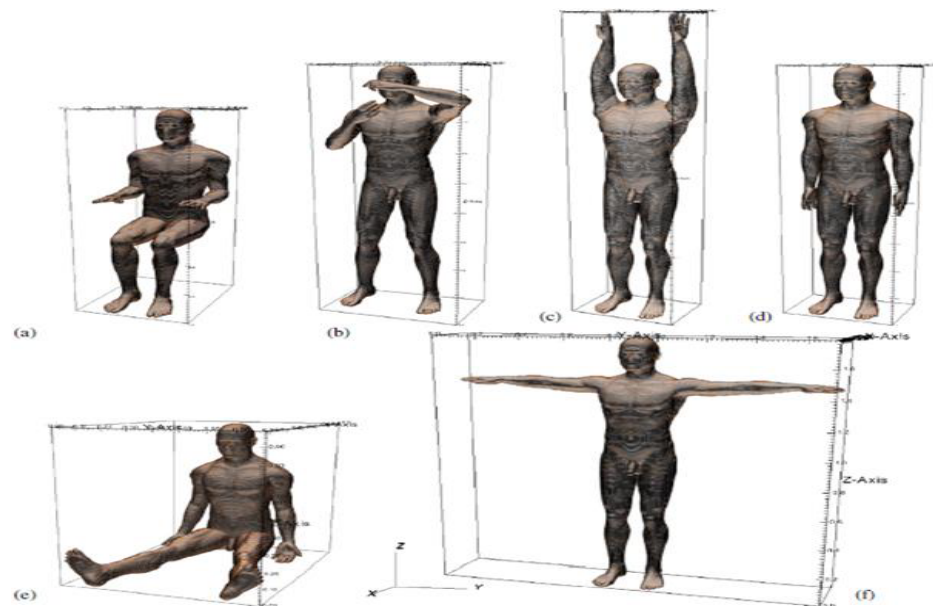

Fig. 1. Posture model used in FDTD a) sit b) examine c) arms up d) standing e) sit on ground f) arms out [18]. 
Before the simulation is performed formally, study the influence of the voxels size and mesh cells to the final results is necessary to perform the convergence calculation. Table 3 shows how whole body current changes with the voxels size and the number of mesh cells when voltage is fixed to be $220 \mathrm{~V}$. Using three $\mathrm{mm}$ or four mm voxel size human body model requires much more mesh cells, and then needs much more memory and hard to do the simulation [19].

Table 3. The number of mesh cell depend on voxel size[19].

\begin{tabular}{|c|c|c|}
\hline Voxel size (mm) & Number of mesh cell & Body current (mA) \\
\hline 8 & $11,476,290$ & 49.3 \\
\hline 6 & $11,674,530$ & 56.3 \\
\hline 5 & $12,072,489$ & 58.5 \\
\hline 4 & $14,479,368$ & 65.5 \\
\hline 3 & $35,123,088$ & 66.6 \\
\hline 2 & $116,385,060$ & 65.8 \\
\hline
\end{tabular}

\subsection{Influence of specific absorption rate (SAR) of the human body using computer simulation technology (CST) microwave studio software for frequency $\mathbf{7 0} \mathrm{mhz}$ under communication system.}

Figure 2 shows the localized SAR (averaged on $10 \mathrm{~g}$ mass) distribution in the human body with sitting and standing posture at $70 \mathrm{MHz}$. For the human body with sitting posture in Figure 2, most of the SAR value is absorbed near different kinds of joints such as ankle, knee, hip joint, wrist, elbow and neck. The area near the nose and the fingers also has a little higher localized SAR. For the human body with standing posture in Figure 3, higher SAR values mainly appear in the legs, especially near the ankle and knee joint [19].
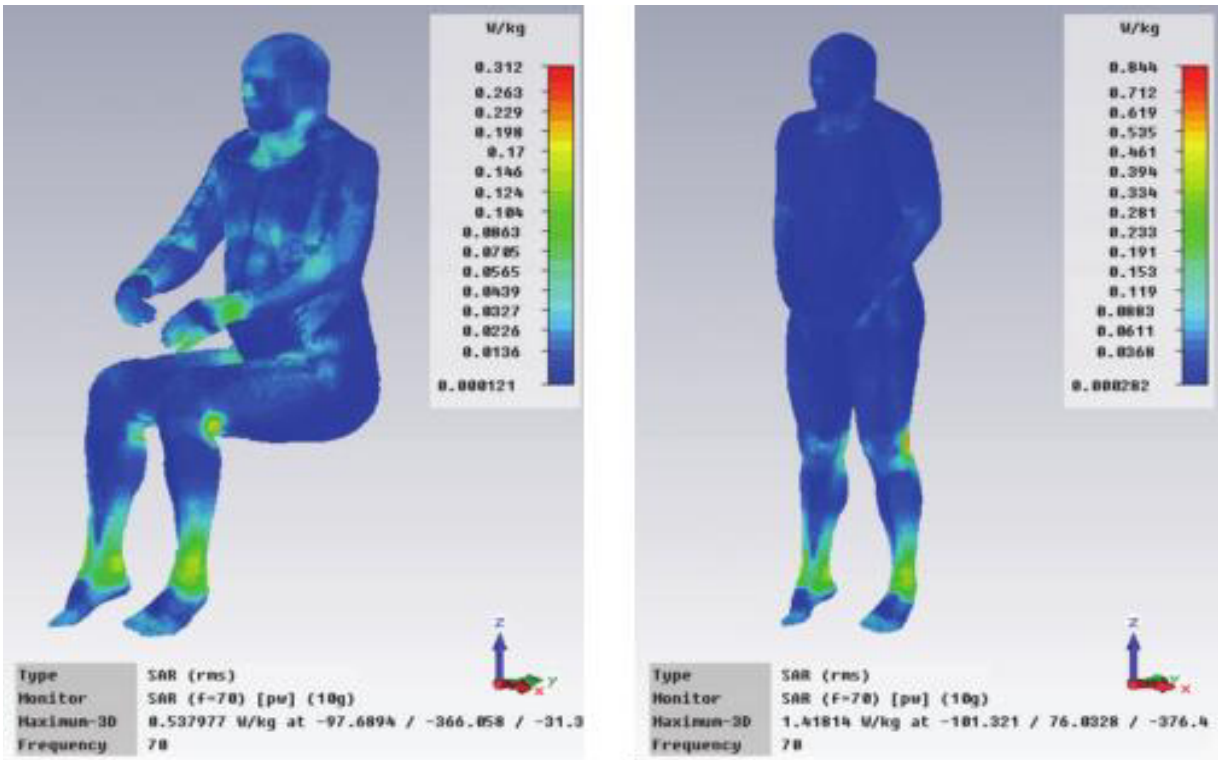

Fig. 2. The SAR distribution in the human body at frequency $70 \mathrm{MHz}$ a) sitting posture b) standing posture[19]. 


\section{Conclusion}

In this simulation, a homogeneous model of man is developed to display the mesh cells value. Based on two designs from the brick block and cylinder block, the cylinder block has higher value of the mesh cells than the brick block. This is because the cylinder block needs detail the voxels size to produce cylinder curve. The smaller of the voxels size value, the higher of the mesh cell value. This project was needed to simulate the specific absorption rate (SAR) to four types of posture. This analysis was done on four types of human position postures which is standing, sitting, arms up and arms out posture. Each posture has different value of SAR based on the size of the mesh cells of the design.

\section{References}

1. P. Akinlolu, A. Kazeem, American Journal of Engineering Research (AJER) 5, 154 (2015)

2. W. headquartes, Legrand power guide, 1, (2009)

3. ICNIRP, Guidelines for limiting exposure to time varying electric, magnetic, and electromagnetic fields (up to 300GHz), (ICNIRP Guidelines, 1998)

4. IEEE-SA Standards Board, 1, (1998)

5. A. Cortel, N. Varsier, V. Dronne, O. Colas, M. F. Wong, E. Nicolas, F. Jacquin, J. Wiart, Proceeding of The International Union of Radio Science (2005)

6. T. Wessapan, S. Srisawatdhisukul, P. Rattanadecho, Int. Commun. Heat Mass, 38, $255(2011)$

7. G.C.R. Melia, Electromagnetic Absorption by the Human Body from 1 to $15 \mathrm{GHz}$, (The University of York, 2013)

8. G.V. Cossio, Giesen, Araya, Tohme, Holmberg, Bressmann, Radiofreq. Electromagn. XXXIII, 81 (2012)

9. A.K.M.F. Hoque, Am. J. Phys. Appl., 1, 104 (2013)

10. Hamzah Asyrani Sulaiman, Mohd Azlishah Othman, Mohamad Zoinol Abidin Abd. Aziz, Mohd Fareq Abd Malek, Theory and Applications of Applied Electromagnetics: APPEIC 2014, (Springer, 2015)

11. M.H. Mat, M.F. Abd Malek, W.G. Whittow, R. Bibb, J. Electromagnet Wave., 29, $514(2015)$

12. ICNIRP, Health Phys., 74, 494 (1998)

13. The World Wide Voltage/Frequency Guide, (http://www.hightechcords.com)

14. Safety Guildelines and Electrical Requirement, Measurement, 429905, 1.

15. P. M. Kanarev, J. Theor. 5, 1 (2004)

16. S. Watanabe, N. Hirose, T. Nagaoka, N. Hatakenaka, Y. Tanaka, M. Takahashi, Y. Suzuki, M. Taki, J. Wang, O. Fujiwara, and Y. Yamanaka, Int. Symp. Electromagn. Compat., 2, 797 (2004)

17. A. Lee, H. Choi, General Assembly and Scientific Symposium, 2011 XXXth URSI, 5 (IEEE, 2011)

18. T. Uusitupa, I. Laakso, S. Ilvonen, K. N. Sar, T. Uusitupa, I. Laakso, S. Ilvonen, and K. Nikoskinen, Phys. Med. Biol., 55, 1157 (2010)

19. J. Gao, Generation of Postured Voxel-based Human Models Used for Electromagnetic Applications, (Technische Universität, Darmstadt, 2012)

20. T.M. Uusitupa, S.A. Ilvonen, I.M. Laakso, K.I. Nikoskinen, Phys. Med. Biol., 53, $445(2008)$ 